\title{
Differential effects of dosing regimen on the safety and efficacy of dasatinib: retrospective exposure- response analysis of a Phase III study
}

This article was published in the following Dove Press journal:

Clinical Pharmacology:Advances and Applications

7 June 2013

Number of times this article has been viewed

Xiaoning Wang'

Amit Roy ${ }^{2}$

Andreas Hochhaus ${ }^{3}$

Hagop M Kantarjian ${ }^{4}$

Tai-Tsang Chen ${ }^{5}$

Neil P Shah ${ }^{6}$

'Discovery Medicine and Clinical Pharmacology, Bristol-Myers Squibb, Lawrenceville, NJ, USA; ${ }^{2}$ Clinical Pharmacology and Pharmacometrics, Research and Development, BristolMyers Squibb, Lawrenceville, NJ, USA; ${ }^{3}$ Hematology/Oncology, Jena University Hospital, Jena, Germany; ${ }^{4}$ Department of Leukemia, Division of Cancer Medicine, The University of Texas MD Anderson Cancer Center, Houston, TX, USA; ${ }^{5}$ Department of Global Biometric Sciences, BristolMyers Squibb, Wallingford, CT, USA; ${ }^{6} \mathrm{Hematology/Oncology,} \mathrm{University} \mathrm{of}$ California, San Francisco School of Medicine, San Francisco, CA, USA

Correspondence: Neil P Shah UCSF-Hematology/Oncology, Suite MI286, Box I270, 505 Parnassus Ave, San Francisco, CA 94I43, USA

$\mathrm{Tel}+\mathrm{I} 4154763303$

Fax +I 4154763726

Email nshah@medicine.ucsf.edu
Purpose: Dasatinib is a prototypic short half-life BCR-ABL1 tyrosine kinase inhibitor. The recommended dose of dasatinib for chronic myeloid leukemia in chronic phase was changed from $70 \mathrm{mg}$ twice daily to $100 \mathrm{mg}$ once daily following a Phase III dose-optimization study. To better understand the superior benefit-risk profile of dasatinib $100 \mathrm{mg}$ once daily, exposure-response was characterized for efficacy (major cytogenetic response) and safety (pleural effusion).

Patients and methods: Dasatinib exposure in patients with chronic myeloid leukemia in chronic phase was determined by population pharmacokinetic analysis of data from seven dasatinib clinical studies ( $N=981)$, including the Phase III dose-optimization study $(n=567)$. Data from the Phase III study were then used to characterize exposure-response relationships for the four dasatinib treatment regimens investigated (100 mg once daily, $50 \mathrm{mg}$ twice daily, $140 \mathrm{mg}$ once daily, and $70 \mathrm{mg}$ twice daily).

Results: Major cytogenetic response was significantly $(P<0.01)$ associated with weighted average steady-state dasatinib plasma concentrations, and pleural effusion was significantly associated with trough concentration. Major cytogenetic response was also significantly associated with maintenance of uninterrupted dosing. The $100 \mathrm{mg}$ once daily arm had the lowest steady-state trough concentration of the four dose arms investigated in the Phase III study, and although this arm also had the lowest weighted average steady-state dasatinib plasma concentration, it had the highest dose maintenance.

Conclusion: Dasatinib dose optimization to $100 \mathrm{mg}$ once daily from $70 \mathrm{mg}$ twice daily significantly minimizes adverse events while maintaining efficacy by exploiting differences in the measures of exposure associated with efficacy and safety.

Keywords: chronic myeloid leukemia, pharmacokinetics, major cytogenetic response, pleural effusion

\section{Introduction}

In 2010, chronic myeloid leukemia (CML) accounted for $11 \%$ of all adult leukemias diagnosed in the United States. ${ }^{1,2} \mathrm{CML}$ is characterized by the fusion of a portion of the $A B L 1$ oncogene on chromosome 9 with the breakpoint cluster region gene $(B C R)$ on chromosome 22 to form the $B C R-A B L 1$ gene. ${ }^{1}$ This oncogene encodes a constitutively active tyrosine kinase protein (BCR-ABL1) that can activate multiple signal transduction pathways affecting hematopoietic cell growth and survival. ${ }^{3}$ BCR-ABL1 tyrosine kinase inhibitors (TKIs) (imatinib, dasatinib, and nilotinib) are currently the mainstays of CML treatment. ${ }^{4}$

Dasatinib is a prototypic short half-life TKI (plasma half-life approximately 4 to 6 hours $)^{5}$ that inhibits BCR-ABL1 with a potency 325 -fold that of imatinib in vitro. 
It is also active against several imatinib-resistant BCRABL1 mutants. ${ }^{6}$ Dasatinib was initially approved for the treatment of adults with Philadelphia chromosome-positive $(\mathrm{Ph}+) \mathrm{CML}$ in chronic, accelerated, or myeloid/lymphoid blast phase (CML-CP, -AP, or -BP, respectively) with resistance or intolerance to prior therapy, including imatinib, or Ph+ acute lymphoblastic leukemia (ALL) with resistance or intolerance to prior therapy. ${ }^{7}$ It has since been approved for the treatment of newly diagnosed adults with $\mathrm{Ph}+\mathrm{CML}-\mathrm{CP}$. Dose and schedule were initially explored in a Phase I doseescalation study in which 84 patients with CML or Ph+ALL that was resistant or intolerant to imatinib received dasatinib (15-240 mg/day) once daily or twice daily. ${ }^{8}$ In this study, the $70 \mathrm{mg}$ twice daily dose showed the most notable rates of major cytogenetic response (MCyR) in both CML-CP (four of six patients) and advanced-phase CML (CML-AP or -BP) (ten of 17 patients). ${ }^{8}$ Based in large part upon the plasma halflife observed in this study, dasatinib $70 \mathrm{mg}$ twice daily was further assessed in five Phase II studies in patients across all phases of CML and Ph+ ALL (the Src/Abl Tyrosine kinase inhibition Activity: Research Trials [START]). ${ }^{9-13}$ These studies served as the basis for approval of the $70 \mathrm{mg}$ twice daily dose in the United States and Europe for Ph+ CML (CP, $\mathrm{AP}$, and $\mathrm{BP}$ ) and $\mathrm{Ph}+\mathrm{ALL}$ in patients intolerant or resistant to imatinib. ${ }^{14,15}$

Retrospective analysis data from the Phase I and Phase II studies in patients with CML suggested that pleural effusion, a key adverse event (AE) associated with dasatinib therapy, is less frequent with once daily dosing compared with twice daily dosing and at doses of $\leq 100 \mathrm{mg} /$ day compared with doses of $\leq 140 \mathrm{mg} /$ day. ${ }^{16}$ Additionally, in two of the Phase II studies of dasatinib $70 \mathrm{mg}$ twice daily in patients with CML$\mathrm{CP}$, the mean total daily dose following dose reductions and interruptions was approximately $100 \mathrm{mg} .{ }^{11,12}$ Based on these data, the dasatinib dose and schedule were prospectively reassessed in an open-label Phase III study of patients with CML-CP ${ }^{17,18}$ in which patients were equally randomized to four dasatinib treatment regimens (100 mg once daily, $50 \mathrm{mg}$ twice daily, $140 \mathrm{mg}$ once daily, and $70 \mathrm{mg}$ twice daily). Similar MCyR rates were observed with dasatinib $100 \mathrm{mg}$ once daily $(n=167)$ and $70 \mathrm{mg}$ twice daily $(\mathrm{n}=168)(59 \%$ and $55 \%$, respectively), whereas dasatinib $100 \mathrm{mg}$ once daily was associated with significantly lower frequencies of grade 3-4 AEs $(30 \%$ versus $48 \%)(P=0.001)$, grade 3-4 thrombocytopenia (22\% versus $37 \%)(P=0.004)$, and any-grade pleural effusion $(7 \%$ versus $16 \%)(P=0.024)$ compared with dasatinib $70 \mathrm{mg}$ twice daily. ${ }^{17}$ Furthermore, fewer patients required dose interruptions and reductions due to toxicity in the $100 \mathrm{mg}$ once daily arm versus the $70 \mathrm{mg}$ twice daily arm. ${ }^{17}$ These data led to a new approved starting dose of $100 \mathrm{mg}$ once daily for patients with CML-CP. ${ }^{19}$

The exposure-response (E-R) relationship was characterized with respect to efficacy (MCyR) and safety (pleural effusion) to better understand and quantify the factors underlying the superior benefit-risk balance of dasatinib $100 \mathrm{mg}$ once daily compared with dasatinib $70 \mathrm{mg}$ twice daily in patients with $\mathrm{Ph}+\mathrm{CML}-\mathrm{CP}$. A previously developed population pharmacokinetic (PPK) model ${ }^{20}$ was updated with data from the Phase III dose-optimization study and applied to determine summary measures of individual dasatinib exposure (both steady-state and time-dependent peak, trough, and time-averaged plasma dasatinib concentrations). These exposure measures were used to characterize dasatinib E-R relationships for efficacy (attainment of MCyR) and safety (pleural effusion incidence). MCyR was selected for the exposure-efficacy response analysis because it was the primary endpoint in the Phase III dose-optimization study. ${ }^{17}$ Pleural effusion was selected for the exposure-safety response analysis because it is the most common fluid retention event reported in patients with CML treated with second-line dasatinib. ${ }^{21}$

\section{Methods Data}

The pharmacokinetics of dasatinib in subjects with CMLCP was described by a PPK model, developed using data from seven open-label clinical studies in patients with CML (one Phase I dose-escalation study of dasatinib 15-180 mg once daily and 25-120 mg twice daily; five Phase II studies of dasatinib $70 \mathrm{mg}$ twice daily [START-A, -B, -C, -L, and -R]; and the Phase III dose-optimization study). ${ }^{8-13,17}$ This model was applied to determine the dasatinib exposure of subjects in the Phase III dose-optimization study from the available, sparse dasatinib concentration measurements in these subjects. The dasatinib E-R for MCyR and pleural effusion was characterized using data from subjects in the Phase III dose-optimization study for whom dasatinib exposure could be determined. The studies included in the PPK and E-R analyses are summarized in Table S1. All studies were approved by the relevant Institutional Review Boards and Independent Ethics Committees of each participating institution and were conducted in accordance with the ethical principles of the Declaration of Helsinki.

The PPK analysis dataset included a total of 6457 dasatinib plasma concentration values from 981 patients with $\mathrm{Ph}+\mathrm{CML}$ (CP, AP, and BP) or Ph+ ALL that was resistant or intolerant to prior therapy from the seven open-label clinical studies. 
Baseline demographic and laboratory measurements were recorded and included in the analysis dataset. A summary of patient demographics and laboratory values included in the PPK model are shown in Table S2.

Efficacy and safety E-R analyses were performed on data from 567 (86\%) of 662 patients with CML-CP treated with dasatinib in the Phase III dose-optimization study for whom dasatinib exposure could be determined. The median age was 55 years (range 18-84), and 47\% (266/567) of patients were male. In total, 34\% of patients (191/567) had a history of cardiac disease. Most patients (74\%, 417/567) were imatinib-resistant, and the others $(26 \%, 150 / 567)$ were imatinib-intolerant.

The primary endpoint for efficacy in the Phase III study was achievement of MCyR after a minimum follow-up of 6 months. Patients were considered to have achieved MCyR if they had $\leq 35 \% \mathrm{Ph}+$ metaphases in bone marrow. ${ }^{17}$ Safety evaluations included pleural effusion incidence and time to first reported pleural effusion (any grade). ${ }^{17,22}$ Chest X-rays were performed at baseline, after 6 months of treatment, and as required for detection or monitoring of pleural effusion.

\section{Analyses}

PPK model

The PPK model was developed by updating a previously developed model ${ }^{20}$ with dasatinib plasma concentration data collected in the Phase III dose-optimization study. Covariate effects (age, gender, race, body weight, body mass index, baseline hepatic and renal laboratory parameters, hemoglobin, and white blood cell count) on PK parameters (clearance and volume of distribution) were evaluated by the likelihood ratio test. Only covariate effects that were both statistically significant $(P<0.001)$ and clinically relevant (defined on the basis of covariate inclusion that resulted in more than a $\pm 20 \%$ parameter change) were retained in the final PPK model. Although no formal adjustment was made for multiplicity, the significance level of $0.1 \%$ was selected in consideration of the multiple parameter-covariate relationships assessed. The model assumed random interindividual variability (IIV) with a log-normal distribution on all structural model parameters. In addition, an interoccasion variability component (IOV) was used to describe the random variability in relative bioavailability within an individual and between dosing occasions. The difference between observed values and the corresponding model-predicted values was described by a log-normal residual error model. A model evaluation was conducted using visual predictive performance checks on the Phase III study data. The observed dasatinib plasma concentration-time data and corresponding fifth, 50th, and 95th percentiles of the model-based predictions were plotted as a graphical assessment.

The PPK model was applied to determine summary measures of dasatinib steady-state exposure for the nominal dose (steady-state peak, trough, and time-averaged plasma dasatinib concentrations $\left[\mathrm{C}_{\max } \mathrm{ss}, \mathrm{C}_{\min } \mathrm{ss}\right.$, and $\mathrm{C}_{\mathrm{avg}} \mathrm{ss}$, respectively]) from the maximum a posteriori estimates of individual PK parameters. $\mathrm{C}_{\text {avg }}$ ss was calculated as the ratio of the steady-state area under the curve to the dosing interval ( 24 hours for once daily and 12 hours for twice daily). The model also was applied to obtain the time-dependent peak, trough, and time-averaged plasma dasatinib concentrations $\left(\mathrm{C}_{\max }, \mathrm{C}_{\min }\right.$, and $\mathrm{C}_{\mathrm{avg}}$, respectively) given the actual dosing history (including dose interruptions and modifications). The model was developed using NONMEM $^{\circledR}$ (version VI, level 1.1; Icon plc, Dublin, Republic of Ireland). Diagnostic graphics, exploratory analyses, and postprocessing of NON$\mathrm{MEM}^{\circledR}$ output were performed using S-PLUS (version 7.0.0 for Linux; TIBCO Software Inc, Palo Alto, CA, USA).

\section{E-R for efficacy: MCyR}

The relationship between dasatinib exposure and the probability of achieving MCyR was described by a logistic regression model. The marginal effect of dasatinib exposure on MCyR was first characterized in a base model, followed by examination of effects from patient covariates in a full model. The following patient covariates were examined: age, gender, imatinib failure status (resistant or intolerant), and duration of dose maintenance (uninterrupted duration as percentage of total treatment duration). The final model was developed by backward elimination of covariate effects from the full model and contained effects from both exposure measures and covariates that had statistically significant effects $(P<0.01)$. Although no formal adjustment was made for multiplicity the significance level of $1 \%$ was selected in consideration of the multiple covariates assessed. The exposure measures $\left(\mathrm{C}_{\max } \mathrm{ss}, \mathrm{C}_{\min } \mathrm{ss}\right.$, and $\left.\mathrm{C}_{\text {avg }} \mathrm{ss}\right)$ for each patient were adjusted to account for dose modification by multiplying these values by the weighted average total daily dose taken by the patient (expressed as a percentage of the nominal dose) to obtain the corresponding weighted average exposures ( $\mathrm{wC}_{\max } \mathrm{ss}, \mathrm{wC}_{\min } \mathrm{ss}$, and $\mathrm{wC}_{\text {avg }} \mathrm{ss}$ ). The weighted average total daily dose of each patient was calculated as the daily dose averaged over the duration of uninterrupted treatment (treatment duration excluding dose interruptions) up to time of MCyR or end of treatment, whichever occurred earlier. The potential effect of dose interruption was assessed with 
respect to dose maintenance $\left(\mathrm{D}_{\mathrm{m}}\right)$ (expressed as the percentage of uninterrupted treatment duration). The final model was evaluated by assessing the agreement between the observed proportion of MCyR and the $90 \%$ model prediction intervals.

\section{E-R for safety: pleural effusion}

The relationship between dasatinib exposure and the time to first occurrence of grade $\geq 1$ pleural effusion was described by a Cox proportional hazards model. The marginal effect of dasatinib exposure on the occurrence of pleural effusion was first characterized in a base model, followed by the examination of effects from patient covariates (age, gender, race, and history of cardiac disease) in a full model. The final model was developed by backward elimination of covariate effects from the full model and contained both exposure measures and covariates with statistically significant effects $(P<0.01)$. Although no formal adjustment was made for multiplicity, the significance level of $1 \%$ was selected in consideration of the multiple covariates assessed. The measures of exposure assessed were $\mathrm{C}_{\max }, \mathrm{C}_{\min }$, and $\mathrm{C}_{\text {avg }}$, and the model was evaluated by comparing the predicted cumulative probability of pleural effusion with that determined by Kaplan-Meier analysis.

\section{Results PPK analysis}

The dasatinib concentration-time data were well-described by a linear two-compartment PPK model. The model was parameterized in terms of plasma and intercompartmental apparent clearances, apparent volumes of distribution in the central and peripheral compartments, and the absorption rate constant (Table 1). The mean terminal half-life was estimated to be 2.93 hours. The variability in relative bioavailability (IIV of $34.6 \%$ and IOV of $37.4 \%$ ) accounted for a larger portion of overall variability in dasatinib exposure than did the variability in the apparent plasma clearance $(28.8 \%)$. None of the covariates examined during model development had statistically significant effects $(P<0.001)$ or clinically relevant effects $(> \pm 20 \%$ effect) on the PK parameters. Therefore, the final PPK model contained no covariate effects. Evaluation of the diagnostic plots, such as the model predictions versus observations, residuals versus model predictions, and residuals versus time, showed that the model described the observed data well, and assumptions about random variability (ie, IIV, IOV, and residual error) were reasonably satisfied (data not shown).

Figure 1 shows the observed and model-predicted median (90\% prediction intervals) of dasatinib plasma concentration versus time for the four arms of the Phase III
Table I Final PPK model parameter estimates

\begin{tabular}{|c|c|c|c|}
\hline $\begin{array}{l}\text { Parameter } \\
\text { (units) }\end{array}$ & Estimate $^{a}$ & $\begin{array}{l}\text { Standard error } \\
\text { (RSE\%) }\end{array}$ & $95 \% \mathrm{Cl}^{\mathrm{b}}$ \\
\hline \multicolumn{4}{|l|}{ Fixed effects } \\
\hline$(\mathrm{CL} / \mathrm{F})_{\mathrm{TV}}(\mathrm{L} / \mathrm{h})$ & 296 & $6.42(2)$ & $283-309$ \\
\hline$(\mathrm{Vc} / \mathrm{F})_{\mathrm{TV}}(\mathrm{L})$ & 1230 & $63.7(5)$ & $1110-1350$ \\
\hline$(\mathrm{Q} / \mathrm{F})_{\mathrm{TV}}(\mathrm{L} / \mathrm{h})$ & 119 & $6.05(5)$ & $|07-| 3 \mid$ \\
\hline$\left(\mathrm{V}_{\mathrm{p}} / \mathrm{F}\right)_{\mathrm{TV}}(\mathrm{L})$ & 1030 & $38.9(4)$ & $954-1110$ \\
\hline$K A_{T V}(I / h)$ & 2.1 & $0.15(7.3)$ & I.8-2.4 \\
\hline \multicolumn{4}{|l|}{ Random effects } \\
\hline$\omega_{\mathrm{CL}}^{2}$ & $0.083(0.29)$ & $0.016(19.6)$ & $0.051-0.114$ \\
\hline$\omega^{2} \mathrm{~V} c$ & $0.730(0.85)$ & $0.073(10.0)$ & $0.587-0.873$ \\
\hline$\omega^{2} \mathrm{KA}$ (fixed) & $1.0(\mathrm{I} .0)$ & - & - \\
\hline$\omega^{2} F_{R}$ & $0.120(0.35)$ & $0.020(16.2)$ & $0.082-0.158$ \\
\hline$\omega^{2} \mathrm{~F}_{\mathrm{R}, \mathrm{IOV}}$ & $0.140(0.37)$ & $0.008(5.6)$ & $0.125-0.155$ \\
\hline$\omega_{C L, v_{c}}^{2}$ & $0.24 I(0.98)$ & $0.03 \mid(\mid 2.8)$ & $0.181-0.301$ \\
\hline \multicolumn{4}{|l|}{ Residual error } \\
\hline$\sigma_{\mathrm{L}}$ & 0.464 & $0.002(0.537)$ & $0.459-0.469$ \\
\hline
\end{tabular}

Notes: ${ }^{a}$ Estimate values in parentheses are standard deviations for estimated variances (first five entries under random effects) and correlation for estimated covariance (last entry under random effects); bootstrap confidence intervals (327 successful out of a total of 500).

Abbreviations: $\mathrm{Cl}$, confidence interval; $(\mathrm{CL} / \mathrm{F})_{\mathrm{TV}}$, apparent clearance; $\mathrm{KA}$, absorption rate constant; PPK, population pharmacokinetics; $(\mathrm{Q} / \mathrm{F})_{\mathrm{TV}}$, apparent intercompartmental clearance; RSE\%, relative standard error as a percentage of the estimate; $(\mathrm{Vc} / \mathrm{F})_{\mathrm{TV}}$, apparent volume of central compartment; $\left(\mathrm{V}_{\mathrm{P}} / \mathrm{F}\right)_{\mathrm{TV}}$, apparent volume of peripheral compartment; $\sigma_{\mathrm{L}}$, standard deviation of log-additive residual error; $\omega_{C L}^{2}$, variance of interindividual variability for apparent clearance; $\omega_{C L, V c}^{2}$, covariance for apparent clearance and apparent volume of central compartment; $\omega^{2} F_{R}$, variance of interindividual variability for relative bioavailability; $\omega^{2} F_{R, I V}$, variance of interoccasion variability for relative bioavailability; $\omega^{2} \mathrm{KA}$, variance of interindividual variability for absorption rate constant; $\omega^{2} \vee c$, variance of interindividual variability for apparent volume of central compartment.

dose-optimization study. Overall, the predicted concentrations corresponded well to the observed profiles from the study. The percentage of observations outside the $90 \%$ prediction intervals was generally less than $10 \%$, suggesting that the model had no systematic bias with respect to the dose or frequency of dasatinib administration.

The PPK-model-predicted exposures for the 567 patients in the Phase III study show that the $\mathrm{C}_{\min }$ ss was lowest for the $100 \mathrm{mg}$ once daily regimen and that the $\mathrm{C}_{\text {avg }}$ ss was similar for the $100 \mathrm{mg}$ once daily and $50 \mathrm{mg}$ twice daily regimens and for the $140 \mathrm{mg}$ once daily and $70 \mathrm{mg}$ twice daily regimens (Table 2). These data showed that for a given daily dose, $\mathrm{C}_{\min } \mathrm{ss}$ tends to be lower for the once daily schedule whereas $\mathrm{C}_{\max } \mathrm{ss}$ tends to be higher. The weighted average exposure measures showed similar trends. These summary measures and their time-dependent correlates were subsequently applied in the dasatinib exposure-efficacy and exposure-safety analyses after adjustments for dose modifications that were appropriate for each analysis, as described in the Methods section.

\section{E-R for efficacy: MCyR}

During treatment, 63\% (358/567) of patients in the Phase III dose-optimization study achieved MCyR. Similar MCyR 


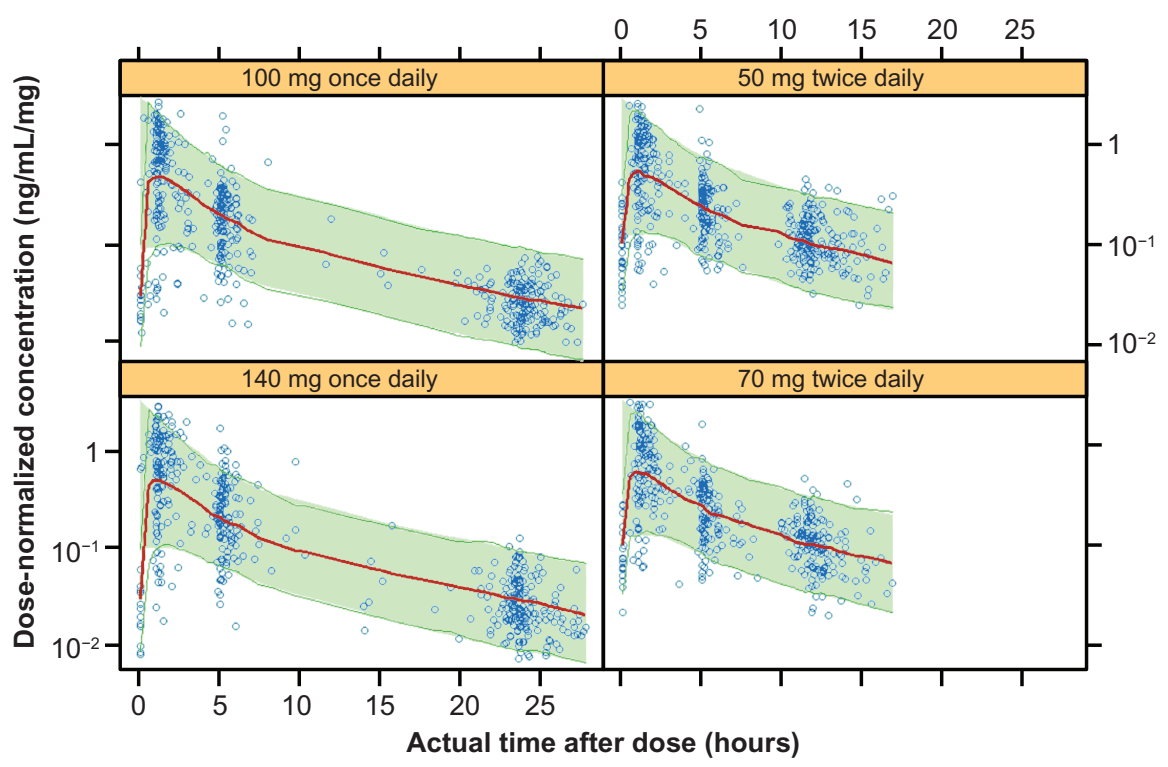

Figure I Observed and predicted median concentrations versus time from previous dose for dasatinib in the Phase III study. ${ }^{17,18}$ Note: Predicted median concentrations have a $90 \%$ prediction interval.

rates were achieved across the four regimens: $64 \%$ (100 mg once daily), $57 \%$ (50 $\mathrm{mg}$ twice daily), $68 \%$ (140 $\mathrm{mg}$ once daily), and 64\% (70 mg twice daily). Of patients included in the analysis dataset, $61 \%(217 / 358)$ of the responders had dose modifications (including dose escalations or interruptions/reductions due to hematologic or nonhematologic toxicity) before achieving MCyR. The predictor variables investigated in the analysis $\left(\mathrm{D}_{\mathrm{m}}, \mathrm{wC}_{\max } \mathrm{ss}, \mathrm{wC}_{\min } \mathrm{ss}\right.$, and $\mathrm{wC}_{\text {avg }} \mathrm{ss}$ ) are summarized in Table 2. Dasatinib $100 \mathrm{mg}$ once daily and $70 \mathrm{mg}$ twice daily had mean reductions of $6 \%$ and $14 \%$ from their nominal daily dose, respectively.

$\mathrm{wC}_{\text {avg }}$ ss was identified as the most significant exposure predictor for achieving MCyR in the base model. The magnitude of the effect of predictor variables assessed in the full model is shown in Figure S1. The effect of gender was not statistically significant and, therefore, not included in the final model. The final logistic regression model (Table 3) indicated that the probability of achieving MCyR in CML-CP patients was significantly greater $(P<0.01)$ with increasing $\mathrm{wC}_{\text {avg }} \mathrm{ss}$ (odds increased 2.11-fold for every doubling of the $\mathrm{wC}_{\text {avg }} \mathrm{ss}$ ), increasing $\mathrm{D}_{\mathrm{m}}$ (odds increased 1.60-fold for every $10 \%$ increase of the $\mathrm{D}_{\mathrm{m}}$ ), and decreasing age (odds decreased $22 \%$ for every decade increase in life). Patients with imatinib-resistant disease were less likely to respond compared with patients with imatinib-intolerant disease (odds ratio $=0.52$ ). Given the definition, $\mathrm{D}_{\mathrm{m}}$ was considered an independent predictor of exposure (correlation coefficient between $\mathrm{wC}_{\text {avg }}$ ss and $\mathrm{D}_{\mathrm{m}}=-0.1$ ).

Table 2 Geometric mean (CV\%) of weighted average daily dose, dose maintenance, and steady-state dasatinib exposures of patients in the Phase III study

\begin{tabular}{|c|c|c|c|c|}
\hline & $\begin{array}{l}100 \mathrm{mg} \text { once daily } \\
n=146\end{array}$ & $\begin{array}{l}50 \mathrm{mg} \text { twice daily } \\
\mathrm{n}=148\end{array}$ & $\begin{array}{l}\mid 40 \mathrm{mg} \text { once daily } \\
\mathrm{n}=|4|\end{array}$ & $\begin{array}{l}70 \mathrm{mg} \text { twice daily } \\
\mathrm{n}=132\end{array}$ \\
\hline wTDD & $94.1(16)$ & $92.0(17)$ & $87.9(16)$ & $85.7(20)$ \\
\hline \multicolumn{5}{|l|}{ (\% nominal) } \\
\hline$D_{m}(\%)$ & $87.8(19)$ & $84.8(20)$ & $84.4(20)$ & $85.1(17)$ \\
\hline$C_{\min } \mathrm{ss}(\mathrm{ng} / \mathrm{mL})$ & $2.61(26)$ & $5.00(24)$ & $3.72(28)$ & $6.71(24)$ \\
\hline $\mathrm{C}_{\max } \mathrm{ss}(\mathrm{ng} / \mathrm{mL})$ & $54.6(56)$ & $32.8(48)$ & $79.7(55)$ & $47.8(46)$ \\
\hline $\mathrm{C}_{\text {avg }} \mathrm{ss}(\mathrm{ng} / \mathrm{mL})$ & I3.5 (35) & I4.3 (3I) & 19.7 (32) & $20.0(3 \mathrm{I})$ \\
\hline $\mathrm{wC}_{\min } \mathrm{ss}(\mathrm{ng} / \mathrm{mL})$ & $2.46(28)$ & $4.59(29)$ & $3.32(31)$ & $5.75(31)$ \\
\hline $\mathrm{wC}_{\max } \mathrm{ss}(\mathrm{ng} / \mathrm{mL})$ & $51.4(58)$ & $30.2(49)$ & $71.2(58)$ & $40.9(52)$ \\
\hline$w_{C_{\text {avg }}} \mathrm{ss}(\mathrm{ng} / \mathrm{mL})$ & $12.7(36)$ & I3.I (33) & $17.5(34)$ & I7.I (37) \\
\hline
\end{tabular}

Abbreviations: $C_{\text {avg }}$ ss, steady-state time-averaged plasma dasatinib concentration; $C_{\max }$ ss, steady-state peak plasma dasatinib concentration; $C_{\text {min }}$ ss, steady-state trough plasma dasatinib concentration; $D_{m}$, percentage dose maintenance duration; $w C_{a v g}$ ss, weighted average steady-state plasma dasatinib concentration; wC $C_{\text {max }} s$, weighted average steady-state peak plasma dasatinib concentration; $w C_{\min } s s$, weighted average steady-state trough plasma dasatinib concentration; wTDD, weighted average total daily dose. 
Table 3 Parameter estimates for dasatinib exposure-efficacy (MCyR) and exposure-safety (pleural effusion) relations

\begin{tabular}{|c|c|c|c|c|}
\hline Predictor $^{a}$ & $\begin{array}{l}\text { Odds ratio } \\
\text { coefficient }^{\mathrm{b}}\end{array}$ & $95 \% \mathrm{Cl}$ & $P$-value & $\begin{array}{l}\text { Odds ratio (5th and 95th } \\
\text { percentiles: median) }\end{array}$ \\
\hline \multicolumn{5}{|c|}{ Logistic regression model for dasatinib exposure-efficacy (MCyR) relation } \\
\hline $\log _{2}\left(w C_{\text {avg }} s s\right)$ & 2.11 & $|.52-2.9|$ & $<0.001$ & $0.43,1.83$ \\
\hline$D_{m} / 10$ & 1.60 & $|.4|-|.8|$ & $<0.001$ & $0.24,2.5 \mathrm{I}$ \\
\hline Age/ 10 & 0.78 & $0.68-0.90$ & 0.001 & $2.00,0.62$ \\
\hline Imatinib status & 0.52 & $0.40-0.65$ & $<0.001$ & - \\
\hline \multicolumn{5}{|c|}{ (resistant versus intolerant) } \\
\hline Predictor & Hazard ratio & $95 \% \mathrm{Cl}$ & $P$-value & Hazard ratio (5th and 95th \\
\hline & coefficient $^{c}$ & & & percentiles: median) \\
\hline \multicolumn{5}{|c|}{ Cox proportional hazard model for dasatinib exposure-safety (pleural effusion) relation } \\
\hline$C_{\text {min }}$ & 1.22 & $1.12-1.33$ & $<0.01$ & $0.63,2.38$ \\
\hline Age/I0 & 2.02 & $1.69-2.43$ & $<0.01$ & $0.13,3.09$ \\
\hline
\end{tabular}

Notes: ${ }^{a} \log _{2}\left(w C_{\text {avg }} s s\right)$ increases by one unit for every doubling of $w C_{\text {avg }} s s ; D_{m} / 10$ increases by one unit for every $10 \%$ increase of $D_{m} ; A g e / / 0$ increases by one unit for every increase of 10 years in age; bincrease in odds for every unit increase in the continuous predictor variable, or odds relative to the reference value of the categorical predictor variable; 'increase in hazard for every unit increase in the continuous predictor variable.

Abbreviations: $\mathrm{Cl}$, confidence interval; $\mathrm{C}_{\text {min }}$, trough plasma dasatinib concentration; $M C y R$, major cytogenetic response; $D_{m}$, percentage dose maintenance duration; wC ${ }_{\text {avg }}$ ss, weighted average steady-state plasma dasatinib concentration.

Figure 2 shows the model-predicted probability (95\% confidence interval [CI]) of MCyR and an evaluation of the model, comparing the observed proportion of MCyR and model-predicted median proportion of MCyR (90\% prediction interval) versus the three continuous predictors $\left(\mathrm{wC}_{\mathrm{avg}} \mathrm{ss}, \mathrm{D}_{\mathrm{m}}\right.$, and age). Each panel shows that patients with imatinib-intolerant disease had higher response probability. The median predictions followed the trend of the relationships, and most of the observed response proportions were covered by the $90 \%$ intervals of the model predictions. The predicted median response rates (with $90 \%$ prediction intervals) for patients treated with dasatinib $100 \mathrm{mg}$ once daily, $50 \mathrm{mg}$ twice daily, $140 \mathrm{mg}$ once daily, and $70 \mathrm{mg}$ twice daily were $53 \%(27 \%-73 \%), 20 \%(7.7 \%-40 \%)$, $60 \%(47 \%-80 \%)$, and $47 \%(27 \%-67 \%)$, respectively. This evaluation indicates that, in general, the model described the relationship between MCyR rate and each predictor reasonably well, although response rate in the $50 \mathrm{mg}$ twice daily group was underpredicted by the model.

\section{E-R for safety: pleural effusion}

Of the 567 patients included in the E-R analysis of the Phase III dose-optimization study, 94 had pleural effusions. Pleural effusion rates were $11.0 \%$ (100 mg once daily), $16.2 \%$ (50 mg twice daily), $17.7 \%$ (140 mg once daily), and $22.0 \%$ (70 mg twice daily). The $\mathrm{C}_{\min }$ was identified as the most significant predictor of pleural effusion in the base model. The magnitudes of covariate effects on the hazard of pleural effusion assessed in the full model are shown in Figure S2. The effects of cardiac disease history, race (Caucasian/ non-Caucasian), and gender were not significant and were removed from the final model. The final model identified age (hazard increased 2.02-fold for every decade increase in life) and $\mathrm{C}_{\min }$ (hazard increased 1.22-fold for every $1 \mathrm{ng} /$ $\mathrm{mL}$ increase in $\mathrm{C}_{\min }$ ) as statistically significant risk factors for pleural effusion $(P<0.01)$ (Table 3$)$. There was no statistically significant $(P<0.01)$ interaction between the $\mathrm{C}_{\text {min }}$ and age, suggesting that age-related variation in $\mathrm{C}_{\min }$ effect was not responsible for age-related variation in response.

Figure 3 illustrates the evaluation of the final model with respect to $\mathrm{C}_{\min }$ and age ( $>55$ years; $\leq 55$ years) by the four dasatinib regimens in the Phase III study. There was generally good agreement between the model-predicted cumulative probability of pleural effusion and the corresponding pleural effusion probability estimates based on Kaplan-Meier analysis (the latter lying within the $90 \%$ model prediction interval), except for a slight overprediction in the $100 \mathrm{mg}$ once daily and $>55$ years age group and a slight underprediction in the $140 \mathrm{mg}$ once daily and $\leq 55$ years age group. As this discrepancy was not consistent across subgroups, it is unlikely to be due to model misspecification. The underprediction noted in the lower age group is most likely a random effect, whereas the deviation in the higher age group may be due to a weak interaction between age and $\mathrm{C}_{\min }$. However, adding the interaction term into the final model did not produce statistical significance.

\section{Discussion}

Optimizing therapeutic strategies for BCR-ABL1 inhibitors is an important area of investigation in CML. ${ }^{17,23-25}$ Given current recommendations for administering BCRABL1 inhibitor therapy indefinitely to patients who are responding to and tolerating these agents, ${ }^{4,26}$ efforts should 
A

Imatinib-intolerant $\triangle$ Imatinib-resistant

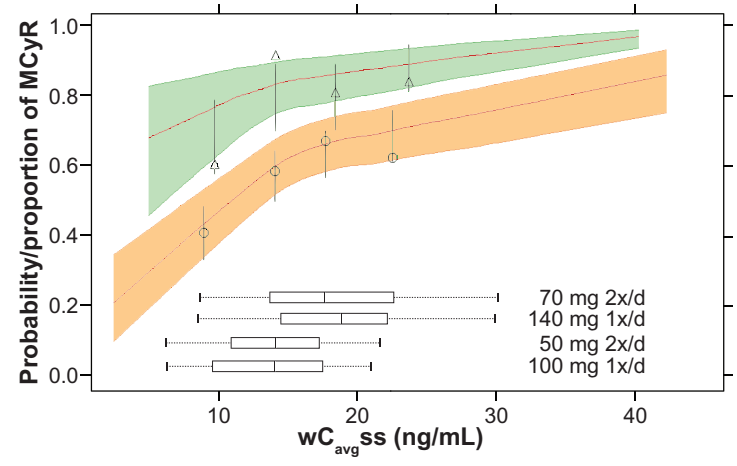

B Imatinib-intolerant Imatinib-resistant

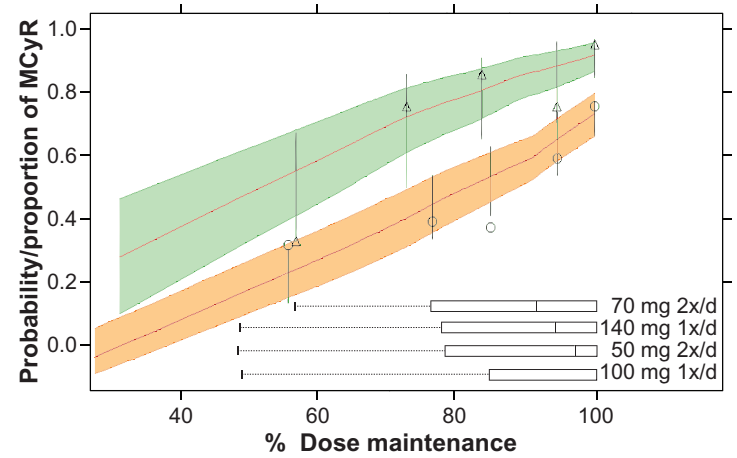

C Imatinib-intolerant $\Delta$

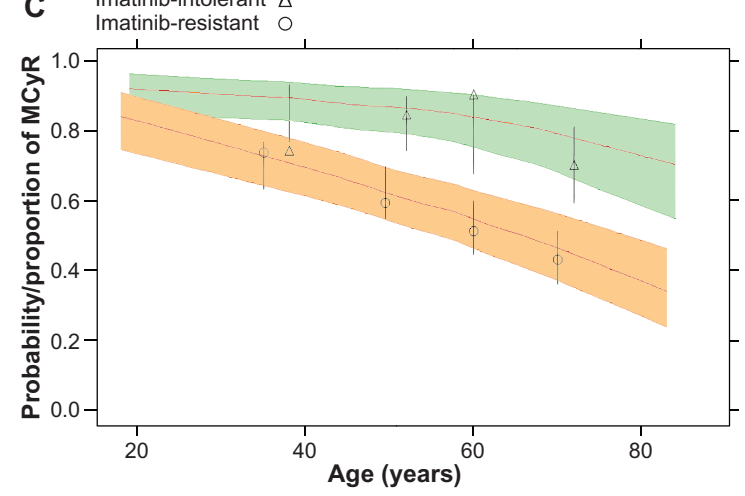

Figure 2 Observed proportion and predicted median proportion/probability of MCyR versus continuous predictors: $(\mathbf{A}) \mathrm{wC}_{\text {avg }} \mathrm{ss},(\mathbf{B})$ dose maintenance, and (C) age.

Notes: The symbols represent the proportion of responders, grouped by quartiles of predictors and plotted at the median for the groups; the centered curves and shaded areas represent median values and $95 \%$ confidence intervals of the modelpredicted response probability, respectively; the vertical bars represent the $90 \%$ model prediction intervals of the MCyR rate, grouped by quartiles of predictors and plotted at the median for the groups; the horizontal box shows the distribution of predictors by treatment arm: the interior bar represents the median, the two ends of the box represent the 25 th and 75 th percentiles, the whiskers represent the fifth and 95 th percentiles; the predicted medians have a $90 \%$ prediction interval.

Abbreviations: $I \times / d$, once daily; $2 \times / d$, twice daily; MCyR, major cytogenetic response; $w_{C_{\text {avg }}} \mathrm{ss}$, weighted average steady-state plasma dasatinib concentration.

be directed toward identifying potentially modifiable risk factors associated with the optimization of response and tolerability. The original dasatinib $70 \mathrm{mg}$ twice daily regimen was selected based on the increased probability of achieving more continuous BCR-ABL1 inhibition and observed clinical responses in Phase I studies. Despite the short halflife of dasatinib, responses were nonetheless observed when the drug was administered once daily. We used data from the Phase III dasatinib dose-optimization study to characterize the E-R relationships for efficacy (MCyR) and safety (pleural effusion) in patients with CML-CP. Our results suggest that it was possible to optimize dasatinib dosage because efficacy and safety were associated with different measures of exposures: achieving MCyR was most closely related to $\mathrm{wC}_{\text {avg }} \mathrm{ss}$, whereas pleural effusion risk was related to $\mathrm{C}_{\min }$. Changing the dosing interval from twice daily to once daily and reducing the daily dose from $140 \mathrm{mg}$ to $100 \mathrm{mg}$ reduced the $\mathrm{C}_{\min }$ and thereby reduced the probability of pleural effusion. Although reduced daily dosing resulted in a nominally lower $\mathrm{C}_{\mathrm{avg}} \mathrm{ss}$, the effect of the reduced exposure on efficacy was ameliorated by fewer dose modifications and interruptions. These results are consistent with the finding that dasatinib $100 \mathrm{mg}$ once daily was associated with similar efficacy and decreased toxicity when compared with the $70 \mathrm{mg}$ twice daily regimen. ${ }^{18}$

The dasatinib concentration-time data for patients enrolled in the Phase III dose-optimization study were welldescribed by a linear two-compartment PPK model with first-order absorption and were consistent with the dasatinib PK from earlier studies. The estimated PK parameters, shown to be time-invariant, agreed reasonably well with previously reported estimates from noncompartmental analyses..$^{20}$ The variability in dasatinib exposure was found to be mainly due to IIV and IOV in bioavailability. In contrast, none of the examined patient covariates appeared to have a clinically relevant effect on dasatinib pharmacokinetics. These findings support the recommendation that dasatinib can be administered without dose adjustment for body weight, age, gender, or race. ${ }^{21}$ Dasatinib exposure appears to be dependent on dosing regimen, as demonstrated by applying the PPK model to the four arms of the Phase III study. Such differentiation in dasatinib exposures provided an opportunity to characterize the $\mathrm{E}-\mathrm{R}$ relationships.

The E-R efficacy analysis results were consistent with previous findings that transient exposure to dasatinib is equivalent to continuous exposure in vitro and that dosing regimens with once daily dasatinib are as effective in achieving rapid and durable clinical responses as twice daily treatment. ${ }^{18,27}$ The most significant predictor of MCyR was $\mathrm{wC}_{\text {avg }}$ ss. Because dasatinib pharmacokinetics are linear, patients treated with once daily and twice daily schedules receiving identical total daily doses should have the same probability of achieving MCyR. This expectation was 


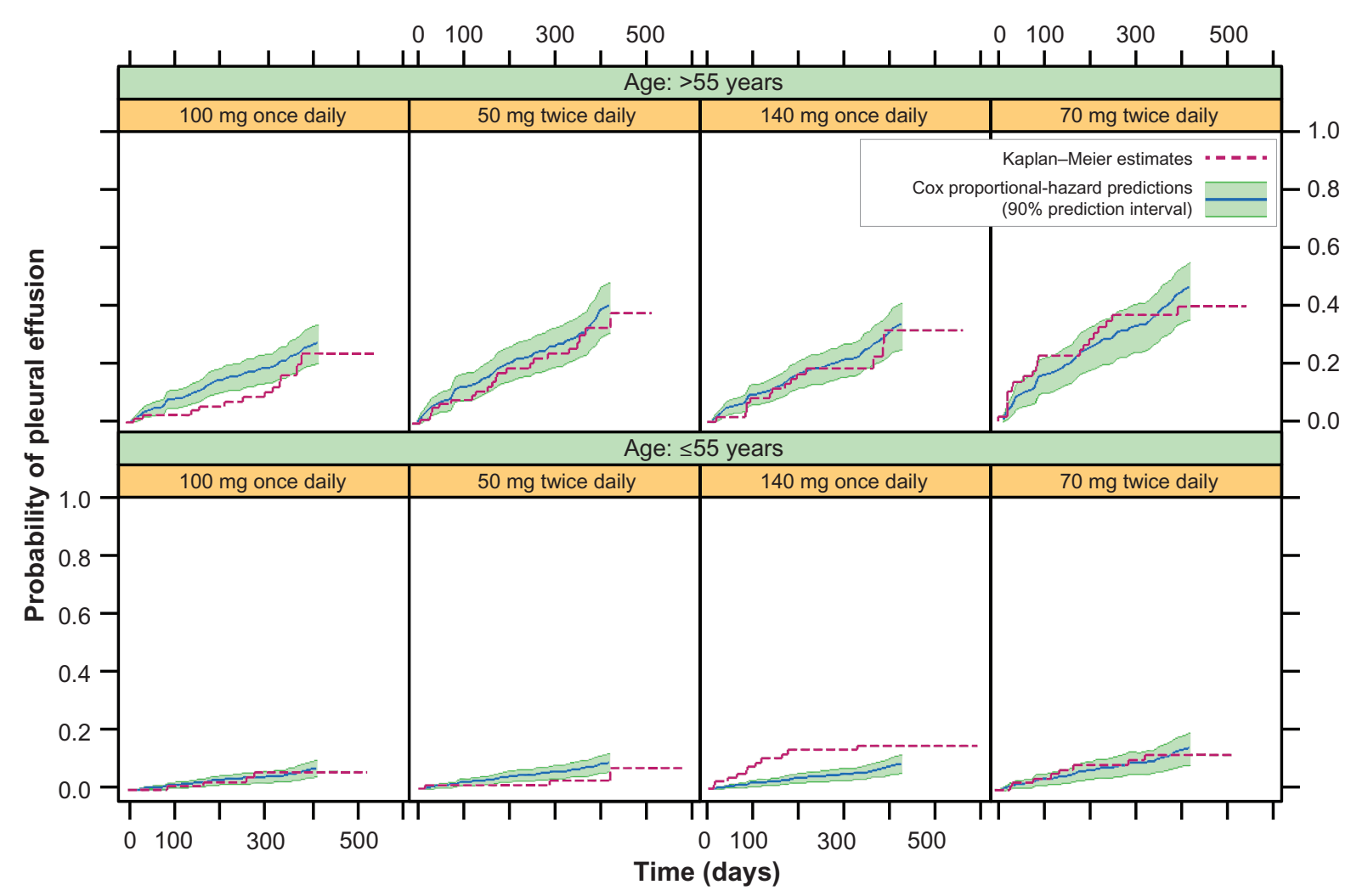

Figure 3 Model-predicted (90\% prediction intervals) and Kaplan-Meier estimated time to pleural effusion by age and dasatinib treatment groups.

consistent with the observed MCyR rates in the Phase III study $(63 \%$ [ $95 \%$ CI, 55.7\%-70.8\%] with $100 \mathrm{mg}$ once daily, $61 \%$ [95\% CI, 53.5\%-68.7\%] with $70 \mathrm{mg}$ twice daily, $63 \%$ [ $95 \% \mathrm{CI}, 55.1 \%-70.2 \%$ ] with $140 \mathrm{mg}$ once daily, and $61 \%$ [95\% CI, 53.5\%-68.7\%] with $50 \mathrm{mg}$ twice daily). ${ }^{18}$ The analysis also identified $\mathrm{D}_{\mathrm{m}}$ as a significant predictor of MCyR, independent of dose modification. Although the predicted transient nature of BCR-ABL1 inhibition with a once daily dosing interval does not negatively affect efficacy, ${ }^{17}$ dose interruptions are expected to have an adverse impact, based on our analysis. Patients treated with dasatinib $100 \mathrm{mg}$ once daily had fewer AEs and dose reductions from the nominal daily dose than those treated with $70 \mathrm{mg}$ twice daily ( $6 \%$ versus $14 \%$, respectively), as well as fewer dose interruptions ( $12 \%$ versus $15 \%$ ). Therefore, maintaining steady dosing may be preferable to higher daily dosing that could lead to dose modifications and interruptions. Analyses for imatinib also revealed that adherence is a critical factor in achieving responses in patients with $\mathrm{CML}^{28,29}$ and that low adherence was likely associated with increased imatinib dose and AEs, although it must be noted that in contrast to dasatinib, imatinib has a prolonged terminal half-life. It is reasonable to believe that any causes of suboptimal adherence, eg, a complex route of administration and drug-food or drug-drug interactions, could negatively impact exposure and hence disease response in real-world settings.

As shown by the data in the Phase III dasatinib study, imatinib-intolerant patients are more likely to achieve MCyR than those with imatinib-resistant disease.${ }^{17}$ Our analysis also suggests that younger patients are more likely to achieve a MCyR than older patients (odds decreased 22\% for every decade increase in life). Gender had no significant association with response in our analysis. The associations of age and gender with response have been studied in patients with CML receiving second-line imatinib, with inconsistent results. ${ }^{30,31}$ In one study, age was a significant predictor of hematologic and cytogenetic response; however, in another, neither age nor gender were independent predictive factors for MCyR. ${ }^{30,31}$

Exposure-safety response analyses performed here identified $\mathrm{C}_{\text {min }}$ as having the strongest association with pleural effusion occurrence. In the Phase III study, the lowest $\mathrm{C}_{\min } \mathrm{ss}$ was achieved with the $100 \mathrm{mg}$ once daily schedule, which had the best benefit-risk balance of the four treatment regimens. ${ }^{17}$ An ongoing Phase II study (OPTIM) is prospectively evaluating the optimization of residual dasatinib plasma levels in patients newly diagnosed with CML-CP. ${ }^{32}$ In the OPTIM study, patients began therapy with dasatinib $100 \mathrm{mg}$ 
once daily, and the $\mathrm{C}_{\max }$ and $\mathrm{C}_{\text {min }}$ were determined after 7 to 10 days. Patients with $\mathrm{C}_{\min }<3 \mathrm{nM}$ continued on $100 \mathrm{mg}$, and those with $\mathrm{C}_{\min }>3 \mathrm{nM}$ were randomly assigned to continue on $100 \mathrm{mg}$ or to have their dose reduced in $20 \mathrm{mg}$ increments until the $\mathrm{C}_{\min }$ (monitored with $\mathrm{C}_{\max }$ in 15-day intervals) decreased to $<3 \mathrm{nM}$. After $\mathrm{C}_{\min }<3 \mathrm{nM}$ was achieved, the $\mathrm{C}_{\min }$ and $\mathrm{C}_{\max }$ were measured every 3 months. After a median follow-up of 7.2 months, the $\mathrm{C}_{\max }$ was found to be associated with response, and the $\mathrm{C}_{\min }$ was associated with fluid retention or pleural effusion. ${ }^{32}$

In addition to the $\mathrm{C}_{\text {min }}$, age was found to be a significant risk factor for pleural effusion. The effect of age is not confounded with exposure, as the PPK analysis showed that exposure to dasatinib did not depend upon age. Furthermore, the Phase III study was well balanced for age across all arms (range of medians, 54-56 years). ${ }^{17}$ Considering the influence of age on pleural effusion risk and the decreased probability of efficacy, the therapeutic window for older patients ( $>55$ years of age) with CML-CP may be narrower relative to younger patients, and these patients may need more intensive monitoring. Cardiac disease history has previously been identified as a risk factor for pleural effusion. ${ }^{16}$ However, in this analysis, cardiac disease history was correlated with age $(\mathrm{R}=0.4)$, and the effect of cardiac disease history was not significant after accounting for the effect of age.

Patients receiving proton pump inhibitors (PPIs) or histamine-2 receptor antagonists were not excluded from this PPK model, although these compounds are likely to reduce dasatinib exposure. ${ }^{21,33}$ For 399 patients with comedication data available, the PPK model estimated the ratio of median exposure between patients who did and did not receive PPIs or histamine- 2 receptor antagonists to be 0.95 for dasatinib $\mathrm{C}_{\min } \mathrm{ss}, 0.76$ for dasatinib $\mathrm{C}_{\max }$ ss, and 0.85 for dasatinib $\mathrm{C}_{\text {avg }} \mathrm{ss}$. This contrasts with results from a drug-drug interaction study in healthy volunteers, in which prior administration of a PPI reduced dasatinib exposure by approximately $60 \% .{ }^{21}$ The more modest effect of PPIs estimated in the current analysis may be due to confounding factors, including polypharmacy and unknown comedication histories and dosing times.

In general, our data show that opportunities for dose regimen optimization exist when the exposure measure most relevant for efficacy is different from that most relevant for safety. Whether this approach may be applied to other BCR-ABL1 TKIs remains controversial. For example, Larson et al have shown the correlation of imatinib $\mathrm{C}_{\text {min }}$ with both response and the occurrence of specific AEs, including fluid retention. ${ }^{34}$ Two more analyses also found an association of imatinib $\mathrm{C}_{\text {min }}$ with clinical response. ${ }^{35,36}$ Yet, data from two other imatinib studies found no correlation between response and $\mathrm{C}_{\min }$; instead, adherence to the standard imatinib dose was critical to achieve response. ${ }^{37,38}$ All these analyses examined $\mathrm{C}_{\min }$ as the only exposure measure, whereas our analysis examined the model-derived $\mathrm{C}_{\max } \mathrm{ss}, \mathrm{C}_{\min } \mathrm{ss}$, and $\mathrm{C}_{\text {avg }}$ ss and selected the most statistically significant predictor. Our analysis also accounted for dose modification. Because of the inconsistencies among these analyses, further research is needed to better understand the E-R relationship of BCR-ABL1 TKIs as a class. It is possible that the efficacy of those agents with prolonged half-lives may correlate better with steady-state concentrations. It also remains to be determined whether the correlations observed in our analysis are applicable to other TKIs with short half-lives.

In conclusion, our analysis shows that dasatinib efficacy and safety were associated with different measures of exposure, $\mathrm{wC}_{\mathrm{avg}} \mathrm{ss}$ and $\mathrm{C}_{\min }$. PPK analyses of the dasatinib Phase III dose-optimization study confirm that the $100 \mathrm{mg}$ once daily schedule was associated with the lowest $\mathrm{C}_{\min } \mathrm{ss}$ value of the studied schedules, corresponding with improved safety compared with dasatinib $70 \mathrm{mg}$ twice daily. ${ }^{18}$ Our analyses highlight opportunities for optimizing BCR-ABL1 TKI dosing regimens and provide insight into the factors that should be considered when selecting an appropriate regimen. By quantifying dasatinib $\mathrm{E}-\mathrm{R}$ relationships, the presented analyses establish a link between dosage regimen and the clinical outcome following drug exposure, providing important insight for optimizing CML therapy.

\section{Acknowledgments}

The authors would like to thank all participating study sites for this Bristol-Myers Squibb-sponsored study. We wish to thank Shruti Agrawal, $\mathrm{PhD}$, for her input and review of the data, and Marc Pfister, MD, and Claude Nicaise, MD, for their insightful comments and critical review of the analyses.

\section{Disclosure}

StemScientific, funded by Bristol-Myers Squibb, provided writing support. The authors did not receive financial compensation from Bristol-Myers Squibb for this analysis. Xiaoning Wang, Amit Roy, and Tai-Tsang Chen are employees of and own stock in Bristol-Myers Squibb. Andreas Hochhaus has received research funding from Bristol-Myers Squibb, Novartis, Pfizer, and Ariad. Hagop Kantarjian has received research funding from Bristol-Myers Squibb, Novartis, and Pfizer and has acted as a consultant for Novartis. Neil Shah is a Leukemia \& Lymphoma Society Scholar in Clinical Research and has acted as a consultant for Bristol-Myers 
Squibb, Novartis, and Ariad. The authors report no other conflicts of interest.

\section{References}

1. Faderl S, Talpaz M, Estrov Z, O’Brien S, Kurzrock R, Kantarjian HM. The biology of chronic myeloid leukemia. N Engl J Med. 1999;341(3):164-172.

2. Jemal A, Siegel R, Xu J, Ward E. Cancer statistics, 2010. CA Cancer J Clin. 2010;60(5):277-300.

3. Sawyers CL. Chronic myeloid leukemia. N Engl J Med. 1999;340(17): $1330-1340$.

4. National Comprehensive Cancer Network. NCCN Clinical Practice Guidelines in Oncology: Chronic Myelogenous Leukemia version 2. 2013. Fort Washington, PA: National Comprehensive Cancer Network; 2012. Available from: http://www.nccn.org/professionals/physician_ gls/f_guidelines.asp. Accessed November 8, 2012.

5. Bristol-Myers Squibb. Dasatinib (BMS-354825) Oncology Drug Advisory Committee (ODAC) Briefing Document NDA 21-986. Wallingford, CT: Bristol-Myers Squibb Company; 2006. Available from: http://www.fda. gov/ohrms/dockets/ac/06/briefing/2006-4220-B1-01BristolMyersSquibbBackground.pdf. Accessed November 8, 2012.

6. O'Hare T, Walters DK, Stoffregen EP, et al. In vitro activity of Bcr-Abl inhibitors AMN107 and BMS-354825 against clinically relevant imatinib-resistant Abl kinase domain mutants. Cancer Res. 2005;65(11):4500-4505.

7. Sprycel ${ }^{\circledR}$ (dasatinib) [summary of product characteristics]. Epernon: Bristol-Myers Squibb Company; 2012.

8. Talpaz M, Shah NP, Kantarjian H, et al. Dasatinib in imatinibresistant Philadelphia chromosome-positive leukemias. $N$ Engl J Med. 2006;354(24):2531-2541.

9. Cortes J, Rousselot P, Kim DW, et al. Dasatinib induces complete hematologic and cytogenetic responses in patients with imatinibresistant or -intolerant chronic myeloid leukemia in blast crisis. Blood. 2007;109(8):3207-3213.

10. Guilhot F, Apperley J, Kim DW, et al. Dasatinib induces significant hematologic and cytogenetic responses in patients with imatinibresistant or -intolerant chronic myeloid leukemia in accelerated phase. Blood. 2007;109(10):4143-4150.

11. Hochhaus A, Kantarjian HM, Baccarani M, et al. Dasatinib induces notable hematologic and cytogenetic responses in chronic-phase chronic myeloid leukemia after failure of imatinib therapy. Blood. 2007;109(6):2303-2309. Erratum in: Blood. 2007;110(5):1438.

12. Kantarjian H, Pasquini R, Hamerschlak N, et al. Dasatinib or high-dose imatinib for chronic-phase chronic myeloid leukemia after failure of first-line imatinib: a randomized phase 2 trial. Blood. 2007;109(12): $5143-5150$.

13. Ottmann O, Dombret H, Martinelli G, et al. Dasatinib induces rapid hematologic and cytogenetic responses in adult patients with Philadelphia chromosome positive acute lymphoblastic leukemia with resistance or intolerance to imatinib: interim results of a phase 2 study. Blood. 2007;110(7):2309-2315.

14. FDA approves dasatinib (Sprycel) for use in the treatment of adults with chronic phase, accelerated phase, or myeloid or lymphoid blast phase chronic myeloid leukemia [webpage on the Internet]. Silver Spring, MD: US Food and Drug Administration; 2006 [updated May 11, 2009]. Available from: http://www.fda.gov/AboutFDA/CentersOffices/Offi ceofMedicalProductsandTobacco/CDER/ucm095630.htm. Accessed November 8, 2012.

15. European Medicines Agency. Sprycel: EPAR - Scientific Discussion. 2006. London: European Medicines Agency; 2006. Available from: http://www.ema.europa.eu/docs/en_GB/document_library/EPAR_-_ Scientific_Discussion/human/000709/WC500056995.pdf. Accessed November 8, 2012.

16. Quintás-Cardama A, Kantarjian H, O'Brien S, et al. Pleural effusion in patients with chronic myelogenous leukemia treated with dasatinib after imatinib failure. J Clin Oncol. 2007;25(25):3908-3914.
17. Shah NP, Kantarjian HM, Kim DW, et al. Intermittent target inhibition with dasatinib $100 \mathrm{mg}$ once daily preserves efficacy and improves tolerability in imatinib-resistant and -intolerant chronicphase chronic myeloid leukemia. J Clin Oncol. 2008;26(19): 3204-3212.

18. Shah NP, Kim DW, Kantarjian H, et al. Potent, transient inhibition of BCR-ABL with dasatinib $100 \mathrm{mg}$ daily achieves rapid and durable cytogenetic responses and high transformation-free survival rates in chronic phase chronic myeloid leukemia patients with resistance, suboptimal response or intolerance to imatinib. Haematologica. 2010;95(2):232-240.

19. Dasatinib (Sprycel) [webpage on the Internet]. Silver Spring, MD: US Food and Drug Administration; 2010 [updated October 29, 2010]. Available from: http://www.fda.gov/AboutFDA/CentersOffices/Offi ceofMedicalProductsandTobacco/CDER/ucm231538.htm. Accessed November 8, 2012.

20. Dai G, Pfister M, Blackwood-Chirchir A, Roy A. Importance of characterizing determinants of variability in exposure: application to dasatinib in subjects with chronic myeloid leukemia. JClin Pharmacol. 2008;48(11):1254-1269.

21. Spryce ${ }^{\circledR}$ (dasatinib) [prescribing information]. Princeton, NJ: Bristol-Myers Squibb Company; 2011.

22. Porkka K, Khoury HJ, Paquette RL, Matloub Y, Sinha R, Cortes JE. Dasatinib $100 \mathrm{mg}$ once daily minimizes the occurrence of pleural effusion in patients with chronic myeloid leukemia in chronic phase and efficacy is unaffected in patients who develop pleural effusion. Cancer. 2010;116(2):377-386.

23. Litzow MR. Imatinib resistance: obstacles and opportunities. Arch Pathol Lab Med. 2006;130(5):669-679.

24. Cortes JE, Baccarani M, Guilhot F, et al. Phase III, randomized, open-label study of daily imatinib mesylate $400 \mathrm{mg}$ versus $800 \mathrm{mg}$ in patients with newly diagnosed, previously untreated chronic myeloid leukemia in chronic phase using molecular end points: tyrosine kinase inhibitor optimization and selectivity study. J Clin Oncol. 2010;28(3): 424-430.

25. Saglio G, Kim DW, Issaragrisil S, et al; ENESTnd Investigators. Nilotinib versus imatinib for newly diagnosed chronic myeloid leukemia. N Engl J Med. 2010;362(24):2251-2259.

26. Baccarani M, Cortes J, Pane F, et al. Chronic myeloid leukemia: an update of concepts and management recommendations of European LeukemiaNet. J Clin Oncol. 2009;27(35):6041-6051.

27. Shah NP, Kasap C, Weier C, et al. Transient potent BCR-ABL inhibition is sufficient to commit chronic myeloid leukemia cells irreversibly to apoptosis. Cancer Cell. 2008;14(6):485-493.

28. Marin D, Bazeos A, Mahon FX, et al. Adherence is the critical factor for achieving molecular responses in patients with chronic myeloid leukemia who achieve complete cytogenetic responses on imatinib. J Clin Oncol. 2010;28(14):2381-2388.

29. Ibrahim AR, Eliasson L, Apperley JF, et al. Poor adherence is the main reason for loss of CCyR and imatinib failure for chronic myeloid leukemia patients on long-term therapy. Blood. 2011;117(14): 3733-3736.

30. Kantarjian H, Sawyers C, Hochhaus A, et al; for the International STI571 CML Study Group. Hematologic and cytogenetic responses to imatinib mesylate in chronic myelogenous leukemia. $N$ Engl J Med. 2002;346(9):645-652.

31. Rosti G, Iacobucci I, Bassi S, et al. Impact of age on the outcome of patients with chronic myeloid leukemia in late chronic phase: results of a phase II study of the GIMEMA CML Working Party. Haematologica. 2007;92(1):101-105.

32. Rousselot P, Boucher S, Etienne G, et al. Pharmacokinetics of dasatinib as a first line therapy in newly diagnosed CML patients (OPTIM dasatinib trial): correlation with safety and response [Abstract]. Blood. 2010;116(21):3432.

33. Eley T, Luo FR, Agrawal S, et al. Phase I study of the effect of gastric acid $\mathrm{pH}$ modulators on the bioavailability of oral dasatinib in healthy subjects. J Clin Pharmacol. 2009;49(6):700-709. 
34. Larson RA, Druker BJ, Guilhot F, et al; IRIS (International Randomized Interferon vs STI571) Study Group. Imatinib pharmacokinetics and its correlation with response and safety in chronic-phase chronic myeloid leukemia: a subanalysis of the IRIS study. Blood. 2008;111(8): 4022-4028.

35. Sakai M, Miyazaki Y, Matsuo E, et al. Long-term efficacy of imatinib in a practical setting is correlated with imatinib trough concentration that is influenced by body size: a report by the Nagasaki CML Study Group. Int J Hematol. 2009;89(3):319-325.

36. Singh N, Kumar L, Meena R, Velpandian T. Drug monitoring of imatinib levels in patients undergoing therapy for chronic myeloid leukaemia: comparing plasma levels of responders and non-responders. Eur J Clin Pharmacol. 2009;65(6):545-549.
37. Forrest DL, Trainor S, Brinkman RR, et al. Cytogenetic and molecular responses to standard-dose imatinib in chronic myeloid leukemia are correlated with Sokal risk scores and duration of therapy but not trough imatinib plasma levels. Leuk Res. 2009;33(2):271-275.

38. Yoshida C, Komeno T, Hori M, et al. Relevance of the daily dose of imatinib mesylate (IM) rather than its trough plasma concentration for achieving deep molecular response in patients with chronic myeloid leukemia [Abstract]. Blood. 2009;114(22):1112. 


\section{Supplementary materials}

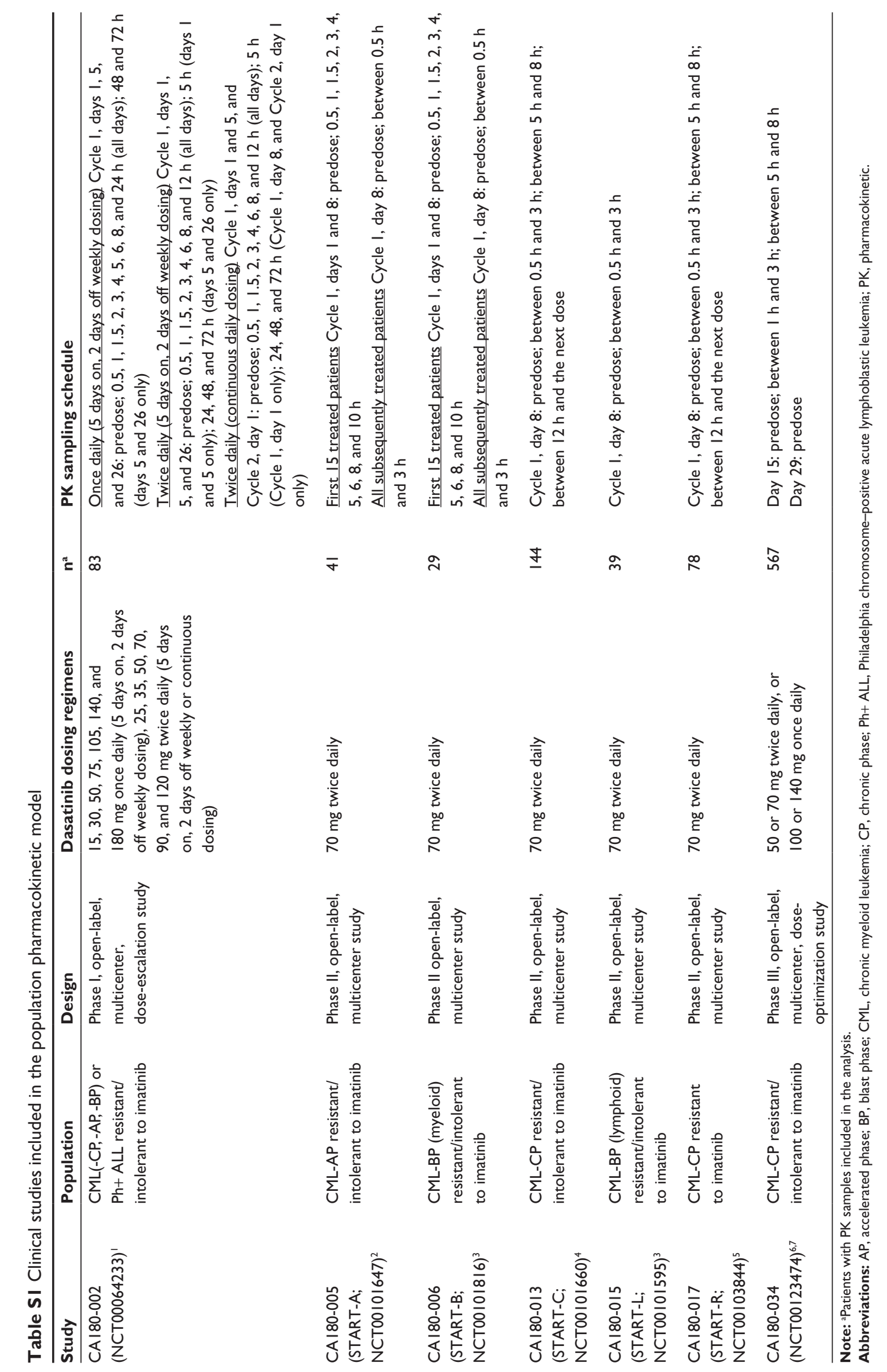


Table S2 Baseline demographic and laboratory values of patients in the population pharmacokinetic analysis

\begin{tabular}{|c|c|c|}
\hline Covariate & $\mathbf{N}$ & Value \\
\hline Median (range) age, years & 981 & $55(15-86)$ \\
\hline Median (range) weight, $\mathrm{kg}$ & 961 & $75(38-180)$ \\
\hline Mean (SD) BMI, kg/m² & 905 & $26.9(5.4)$ \\
\hline Male/female, n (\%) & 981 & $484(49) / 497(5 I)$ \\
\hline Race, n (\%) & 981 & \\
\hline Caucasian & & $816(83)$ \\
\hline Asian & & $83(8)$ \\
\hline Black/African American & & $49(5)$ \\
\hline Other & & $30(3)$ \\
\hline Unknown & & $3(0.3)$ \\
\hline Mean (SD) ALT, IU/L & 944 & $26.2(19.3)$ \\
\hline Mean (SD) AST, IU/L & 946 & $27.7(16.8)$ \\
\hline Mean (SD) creatinine clearance, $\mathrm{mL} / \mathrm{min}$ & 926 & $93.8(35.4)$ \\
\hline Mean (SD) baseline hemoglobin, g/dL & 954 & II.8 (2.0) \\
\hline Mean (SD) baseline WBC count, $\times 10^{3}$ cells $/ \mu \mathrm{L}$ & 959 & $20.6(30.7)$ \\
\hline
\end{tabular}

Abbreviations: ALT, alanine aminotransferase; AST, aspartate aminotransferase; BMI, body mass index; SD, standard deviation; WBC, white blood cell.

Continuous reference

(5th-95th percentile)

categorical comparator: reference

Response to imatinib resistant: intolerant

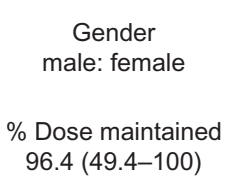

$$
\begin{gathered}
\text { Age (years) } \\
55(28-75)
\end{gathered}
$$

$\mathrm{wC}_{\text {avg }} \mathrm{ss}(\mathrm{ng} / \mathrm{mL})$

$15.8(6.97-27)$

$\begin{array}{lllllllll}0.05 & 0.25 & 0.50 & 1.00 & 2.00 & 4.00 & 8.00\end{array}$

Odds ratio relative to reference value of covariate

Figure SI Estimated odds ratios of the full logistic regression model of MCyR. Notes: The outside edges of the open boxes represent the estimated odds ratio at the fifth percentile of continuous predictor values; the open circles represent odds relative to the reference value of the categorical predictor variable; the outside edges of the shaded boxes represent the estimated odds ratio at the 95th percentile of continuous covariate values; the horizontal lines represent the $95 \%$ confidence intervals of estimated effects.

Abbreviations: MCyR, major cytogenetic response; $w_{\text {avg }}$ ss, weighted average steady-state plasma dasatinib concentration.

Clinical Pharmacology: Advances and Applications

\section{Publish your work in this journal}

Clinical Pharmacology: Advances and Applications is an international, peer-reviewed, open access journal publishing original research, reports, reviews and commentaries on all areas of drug experience in humans. The manuscript management system is completely online and includes a very quick and fair peer-review system, which is all easy to use.

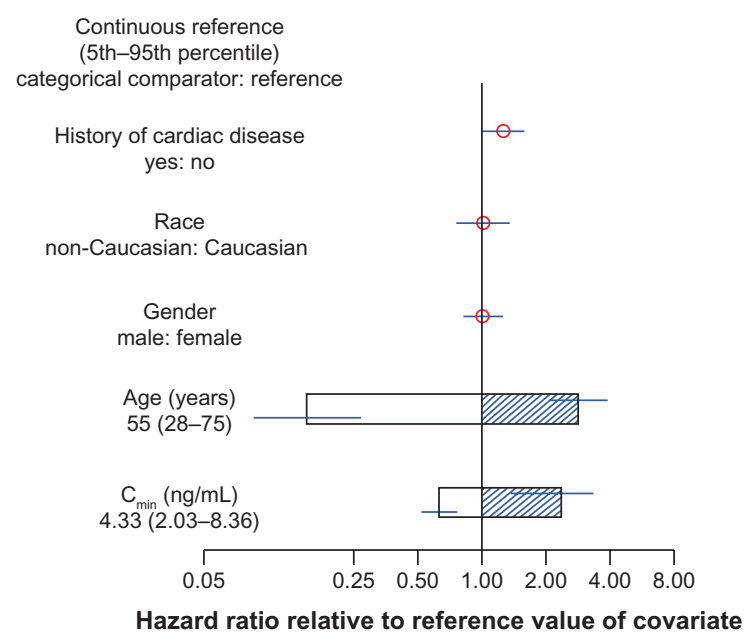

Figure S2 Estimated hazard ratios of the full Cox proportional hazards model of pleural effusion.

Notes: The outside edges of the open boxes represent the estimated hazard ratio at the fifth percentile of continuous predictor values; the open circles represent hazard relative to the reference value of the categorical predictor variable; the outside edges of the shaded boxes represent the estimated hazard ratio at the 95th percentile of continuous covariate values; the horizontal lines represent the $95 \%$ confidence intervals of estimated effects.

Abbreviation: $C_{\text {min }}$, trough plasma dasatinib concentration.

\section{References}

1. Talpaz M, Shah NP, Kantarjian H, et al. Dasatinib in imatinib-resistant Philadelphia chromosome-positive leukemias. N Engl J Med. 2006; 354(24):2531-2541.

2. Guilhot F, Apperley J, Kim DW, et al. Dasatinib induces significant hematologic and cytogenetic responses in patients with imatinib-resistant or -intolerant chronic myeloid leukemia in accelerated phase. Blood. 2007;109(10):4143-4150.

3. Cortes J, Rousselot P, Kim DW, et al. Dasatinib induces complete hematologic and cytogenetic responses in patients with imatinibresistant or -intolerant chronic myeloid leukemia in blast crisis. Blood. 2007;109(8):3207-3213.

4. Hochhaus A, Kantarjian HM, Baccarani M, et al. Dasatinib induces notable hematologic and cytogenetic responses in chronic-phase chronic myeloid leukemia after failure of imatinib therapy. Blood. 2007;109(6):2303-2309. Erratum in: Blood. 2007;110(5):1438.

5. Kantarjian H, Pasquini R, Hamerschlak N, et al. Dasatinib or high-dose imatinib for chronic-phase chronic myeloid leukemia after failure of firstline imatinib: a randomized phase 2 trial. Blood. 2007;109(12):5143-5150.

6. Shah NP, Kantarjian HM, Kim DW, et al. Intermittent target inhibition with dasatinib $100 \mathrm{mg}$ once daily preserves efficacy and improves tolerability in imatinib-resistant and -intolerant chronic-phase chronic myeloid leukemia. J Clin Oncol. 2008;26(19):3204-3212.

7. Shah NP, Kim DW, Kantarjian H, et al. Potent, transient inhibition of BCR$\mathrm{ABL}$ with dasatinib $100 \mathrm{mg}$ daily achieves rapid and durable cytogenetic responses and high transformation-free survival rates in chronic phase chronic myeloid leukemia patients with resistance, suboptimal response or intolerance to imatinib. Haematologica. 2010;95(2):232-240.

\section{Dovepress}

Visit http://www.dovepress.com/testimonials.php to read real quotes from published authors. 